\title{
Galactic anticenter CO survey
}

\author{
I. Area $l=178^{\circ}$ to $186^{\circ}, b=3.5$ to $6^{\circ}$ \\ Y. Lee, J.H. Jung, H.S. Chung, H.-G. Kim, Y.-S. Park, H.R. Kim, B.-G. Kim, J. Kim, and S.-T. Han \\ Korea Astronomy Observatory, Taeduk Radio Astronomy Observatory, San 36-1 Hwaam-dong, Yusung-gu, Taejon \\ 305-348, Korea \\ e-mail: yulee@hae.issa.re.kr
}

Received July 2, 1998; accepted April 09, 1999

\begin{abstract}
We have mapped a 17 square-degree section $(l, b)=\left(178^{\circ} 0 \sim 186^{\circ} 0,3.5 \sim 6.0\right)$ of the Galactic Anticenter region in the ${ }^{12} \mathrm{CO} J=1-0$ line using the 3 -mm SIS receiver on the $14-\mathrm{m}$ telescope at the Taeduk Radio Astronomy Observatory. A total of 7000 spectra has been obtained with a grid spacing of $3^{\prime}$. The results of the observations are presented in the form of $l-b$ and $v-b$ contour maps. Molecular emission of the mapped area is found to be very extended and matches with the FIR emission boundary. The radial velocity of the molecular gas is found to be within the range of $v_{\mathrm{LSR}}=-25 \sim$ $+10 \mathrm{~km} \mathrm{~s}^{-1}$. We also found several small clouds located at $l=180^{\circ}, b=5^{\circ} \sim 6^{\circ}$ having $v_{\mathrm{LSR}}=-20 \mathrm{~km} \mathrm{~s}^{-1}$, which is quite anomalous in this direction. We identified 30 individual clouds within the mapped region with an arbitrary threshold temperature using a cloud identification code. Twelve subclouds were also identified from the largest cloud with a higher threshold temperature. The ratio of dust emission to CO integrated intensity for the Galactic Anticenter region is found to be similar to that of the dark clouds in solar neighborhood, and much less than that of giant molecular clouds, implying that the heating source within the clouds is minimal.
\end{abstract}

Key words: ISM: clouds — ISM: abundance — ISM: dust — ISM: HiI regions

\section{Introduction}

Surveying the Galactic plane for molecular gas has been pursued since the carbon monoxide $2.7-\mathrm{mm}$ line was detected about three decades ago. There were several $\mathrm{CO}$

Send offprint requests to: Y. Lee (ylee@cfa.harvard.edu), visiting scientist at Harvard-Smithsonian Center for Astrophysics, 60 Garden Street, MS-78, Cambridge, MA 02138, U.S.A. surveys toward the Galactic plane (Sanders et al. 1986; Dame et al. 1987; and see Combes 1991 for a review), and substantial information on the distribution and largescale dynamics of molecular gas (and determination of the Galactic rotation curve) has been derived from these surveys. The ${ }^{12} \mathrm{CO}(J=1-0)$ observations by the Columbia University group summarized in Dame et al. (1987) comprise one of the most extensive surveys for molecular clouds, encompassing $30 \%$ of the whole sky along the Galactic plane with an angular resolution of 0.5. The Massachusetts-Stony Brook Galactic plane survey was conducted with a higher resolution of $3^{\prime}-6^{\prime}$, toward the first Galactic quadrant, but was confined within the $\pm 1^{\circ}$ of the Galactic plane (Sanders et al. 1986). Since then, molecular clouds in the inner Galaxy region have frequently been studied, as molecular gas is largely confined to the Molecular Ring in our Galaxy (Scoville et al. 1987; Solomon et al. 1987).

In contrast to investigation inside the solar circle, the molecular gas in the outer Galaxy has been studied in less detail, although there is no confusion in distinguishing foreground from background clouds in the outer Galaxy. Excepting the Columbia survey which was made with a coarse resolution, the first systematic study of molecular clouds in the outer Galaxy was conducted by Mead \& Kutner (1988). Recently, more systematic surveys have been reported on specific regions in the outer Galaxy (Carpenter et al. 1995; Heyer et al. 1998). May et al. (1993, 1997) have analyzed the large-scale physical properties of molecular gas in the outer Galaxy, especially in the third quadrant. A group at Nagoya University has been surveying the outer Galaxy in ${ }^{13} \mathrm{CO}(J=1-0)$, focusing on the dense regions of molecular clouds and investigating star forming activity (Dobashi et al. 1994, 1996; Yonekura et al. 1997; Kawamura et al. 1998). In addition, there were several case studies toward the outer Galaxy molecular clouds; de Geus et al. (1993) reported that there are 


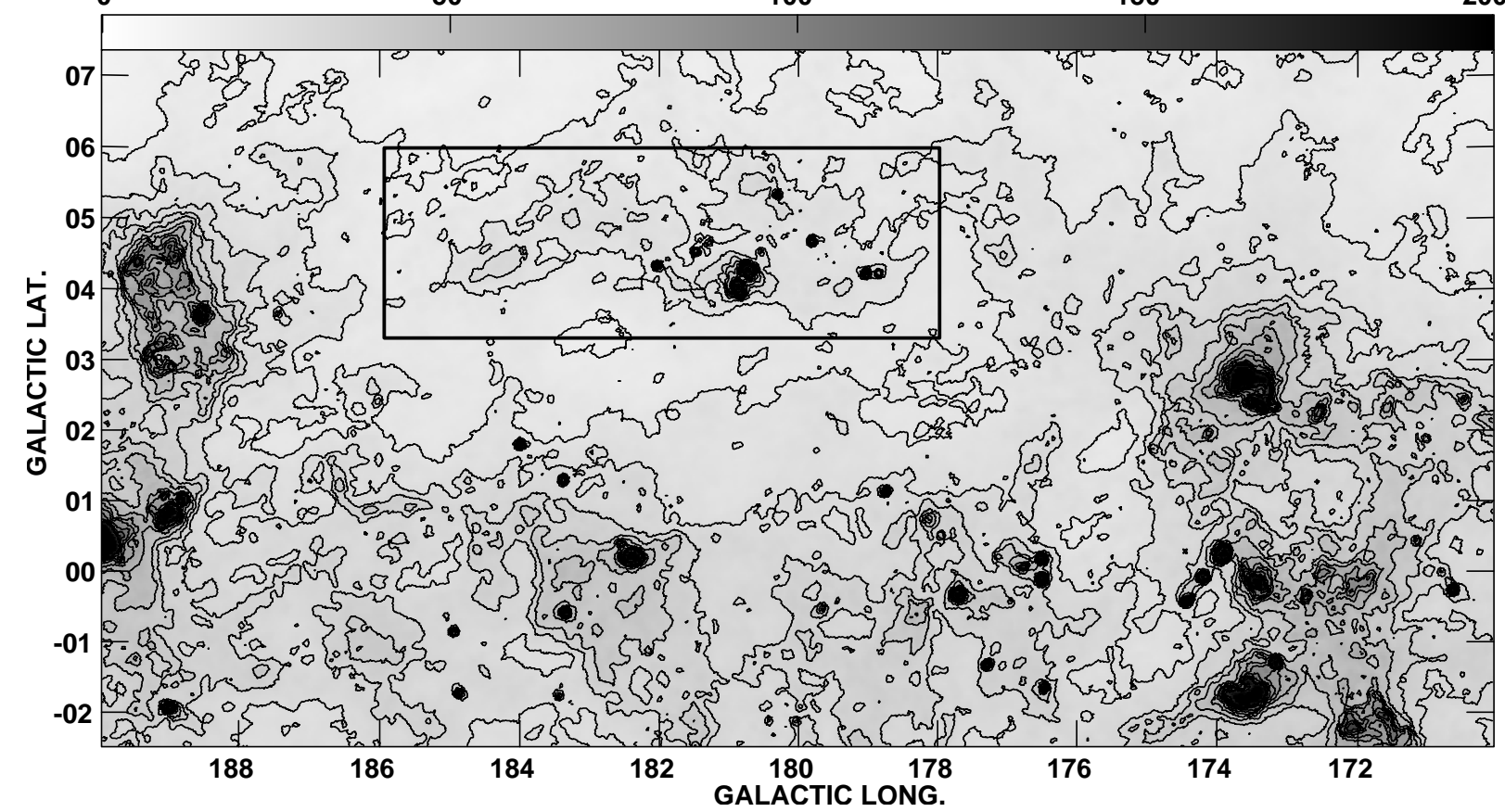

Fig. 1. The $100 \mu \mathrm{m}$ ISSA image of a $20^{\circ} \times 10^{\circ}$ region centered on the Galactic Anticenter. The location of our mapped area is marked with the solid box. The lowest contour level is $10 \mathrm{MJy} \mathrm{sr}^{-1}$, and the increment between levels is $5 \mathrm{MJy} \mathrm{sr}^{-1}$. The grey scale ranges from 1 to $200 \mathrm{MJy} \mathrm{sr}^{-1}$

molecular clouds associated with star forming activity even in the far outer Galaxy $(d=21 \mathrm{kpc}$ and $28 \mathrm{kpc})$ beyond the known optical disk. Brand \& Wouterloot (1994, 1995) focused on the luminously embedded star-forming regions of several molecular clouds in the far outer Galaxy. They showed that star forming clouds are distributed up to $R=20 \mathrm{kpc}$. However, all these CO observations are biased towards the warmest material, and may underestimate, or even miss the colder molecular gas. In fact, Lequeux et al. (1993) found evidence for the existence of significant amounts of cold molecular gas in the outer Galaxy. Digel et al. (1994) also reported that there are several molecular clouds in the extreme outer Galaxy $(18-28 \mathrm{kpc})$ which are much colder than the clouds in the solar neighborhood, and that the metallicity in the far outer Galaxy is so uncertain that the mass calibrating ratio could differ from the local value (see also Mead \& Kutner 1988; Digel et al. 1990). Digel et al. (1996) showed that the clouds in the Perseus arm are systematically less luminous in $\mathrm{CO}$ by a factor of 3.5 than those in the local arm. In their $\mathrm{CO}(2-1) / \mathrm{CO}(1-0)$ study, Usuda et al. (1998) also demonstrated that the intensity of CO emission per unit mass in the outer Galaxy is lower than that in the inner Galaxy. Along with the bright star-forming molecular clouds, the colder population is surely assumed to exist in the outer Galaxy, and more observations are required for investigating further analysis of physical properties of the outer Galaxy molecular clouds.

In contrast to other regions of the Galactic plane, the exact Galactic Anticenter is the least studied region as the conventional velocity-to-distance transformation is of no use because of lack of kinematic resolution. Moreover, the distribution of the molecular gas had been found to be relatively weak. The Columbia survey (Dame et al. 1987) covered the entire Galactic plane, but only samll part of the Galactic Anticenter region was discussed by Huang (1986), mainly focusing on supernovae, including Simeiz 147. In addition, the resolution of Columbia survey (8.7 beamwidth) was not adequate to determine the structure of the clouds, and no higher resolution observations in any molecular species have been published except Kawamura et al. (1998)'s recent ${ }^{13} \mathrm{CO}$ survey with $8^{\prime}$ grid spacing.

In this paper we present the first results of higher resolution ${ }^{12} \mathrm{CO}$ observations of the Galactic Anticenter region, mainly focusing on the distribution of the molecular gas, as a first step in a statistical study of the clouds properties and star forming activity. Complete analysis of statistical properties will be presented in forthcoming paper. Observations, far-infrared (FIR) data and associated objects within the selected region are described in Sect. 2, while in Sect. 3 observational results and spatial-velocity maps are presented. The cloud identification method is briefly explained in Sect. 4 . In Sect. 5 we delineate some highlights of the observational results, and discuss the correlation between $\mathrm{CO}$ emission and dust emission in Sect. 6 . We summarize our results in the final section. 


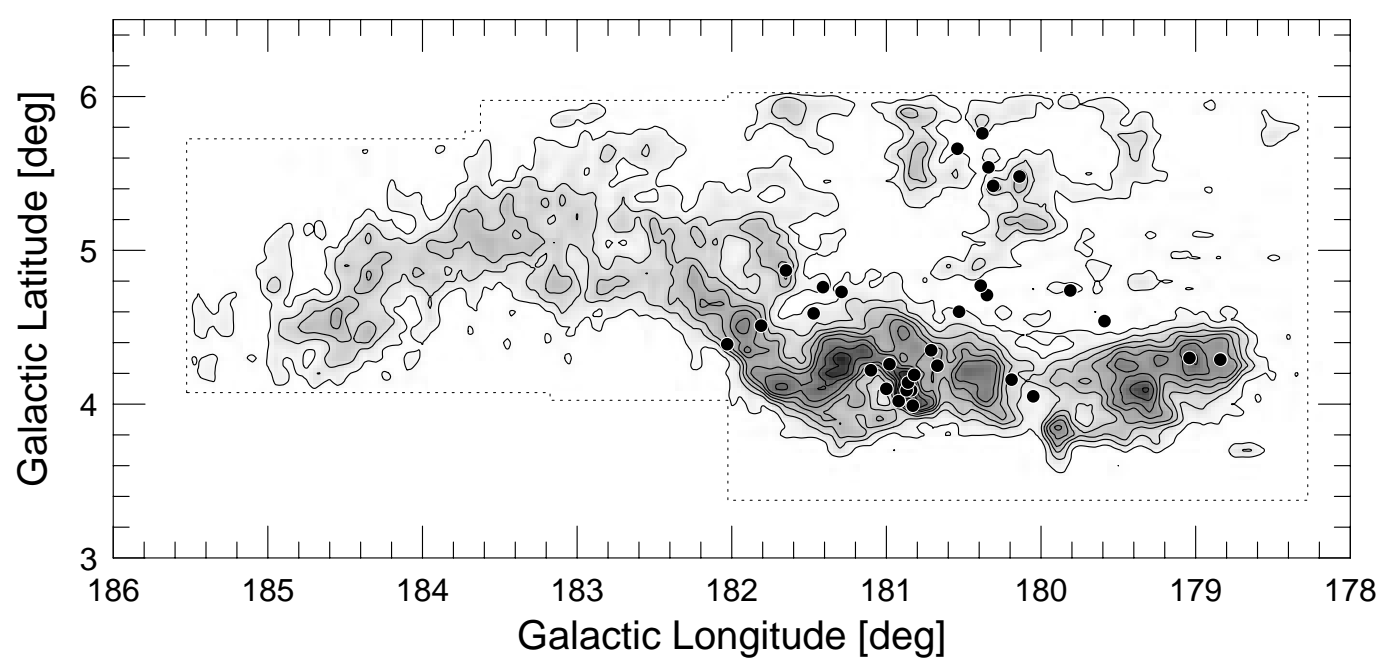

Fig. 2. ${ }^{12} \mathrm{CO}$ integrated intensity map. The grey scale range is 4 to $40 \mathrm{~K} \mathrm{~km} \mathrm{~s}^{-1}$. The lowest contour level is $5 \mathrm{~K} \mathrm{~km} \mathrm{~s}^{-1}$ and the increment between levels is $3 \mathrm{~K} \mathrm{~km} \mathrm{~s}^{-1}$. The dotted box encloses mapped area. The filled circles represents IRAS point sources with $S_{60}>1$ Jy and $S_{100}>S_{60}$

\section{Data taking}

\subsection{Infrared data and associated objects}

To examine the dust emission distribution of the region, we have acquired a wide area $\left(30^{\circ} \times 20^{\circ}\right)$ IRAS Sky Survey Atlas (ISSA) image of the IRAS $100 \mu \mathrm{m}$ band centered on the exact Galactic Anticenter position of $(l, b)$ $=\left(180^{\circ}, 0^{\circ}\right)$ from the Infrared Processing and Analysis Center (IPAC) using Skyview Virtual Observatory (http://skyview.gsfc.nasa.gov/skyview.html). In Fig. 1 we present a $20^{\circ} \times 10^{\circ}$ subregion of the original area obtained. In this figure the strong dust emission around $l=170^{\circ}$ to $174^{\circ}$ traces several Hil regions, including Sh 229, Sh 235, and Sh 236, and many dark clouds. Another strong dust emission region located at $l=187^{\circ}$ to $190^{\circ}$ and beyond is the Gem OB1 giant molecular cloud complex, which was studied extensively by Carpenter et al. (1995). We selected the area indicated with a solid-line box in Fig. 1 as our first target region of the Galactic Anticenter CO Survey (GACCOS) since its dust emission feature is fairly isolated from other brighter emission regions. In addition, we have acquired IRAS point sources from the IRAS Point Source Catalog. Only those IRAS point sources with conspicuous flux (with $S_{60}>1 \mathrm{Jy}$ and $\left.S_{100}>S_{60}\right)$ were collected.

Several molecular clouds are located within the selected region, including 4 Lynds dark clouds (Lynds 1550, Lynds 1555, Lynds 1557, and Lynds 1560). Three Lynds bright nebulae (LBN 823, LBN 824, and LBN 825 ) and only one HiI region Sh 241 (Sharpless 1959) are present. Two reflection nebulae, DG85, DG86 (Dorschner \& Gürtler 1964) are also present, and DG86 (also known as vdB65; van den Bergh 1966) is located close to Sh 241. Overall, compared to the inner Galaxy region, the selected region seems to be quiet, except for the only HII region Sh 241.

\section{2. $C O$ observations}

We have mapped a $17 \mathrm{deg}^{2}$ section $(l, b)=(178.0 \sim$ $\left.186^{\circ} 0,3.5 \sim 6.0\right)$ in ${ }^{12} \mathrm{CO} J=1-0$ using the $3-\mathrm{mm}$ SIS receiver on the Taeduk Radio Astronomy Observatory (TRAO) 14-m telescope. The SIS receiver was developed at TRAO and has been operating since summer 1995 (Han et al. 1995). The beam size (FWHM) is $47^{\prime \prime}$ and the grid spacing of the map is $3^{\prime}$. We mainly used a $250-\mathrm{kHz}$ filterbank, which covers a velocity range of $170 \mathrm{~km} \mathrm{~s}^{-1}$ with a resolution of $0.65 \mathrm{~km} \mathrm{~s}^{-1}$. In addition, we also used $1 \mathrm{MHz}$ filterbank, which covers a velocity coverage of $650 \mathrm{~km} \mathrm{~s}^{-1}$ with a resolution of $2.6 \mathrm{~km} \mathrm{~s}^{-1}$, providing a large velocity range in order to search for any detectable emission at other velocities. The filterbanks were centered at $v_{\mathrm{LSR}}=0 \mathrm{~km} \mathrm{~s}^{-1}$ for all observations.

All observations were made by position switching between observed positions and reference positions which were carefully selected to be free of $\mathrm{CO}$ emission. Each reference observation was shared among 2 to 6 map positions, depending on the sky stability. Calibration was accomplished by frequently observing an ambient temperature load. All antenna temperatures quoted are corrected for atmospheric extinction, and for the forward spillover and scattering losses of the antenna and radome $\left(\eta_{\mathrm{fss}}=\right.$ 0.63 at $115 \mathrm{GHz}$ ), and are therefore on the $T_{\mathrm{R}}^{*}$ temperature scale defined by Kutner \& Ulich (1981). The system temperature varied between $700 \mathrm{~K}$ and $1000 \mathrm{~K}$ depending on the elevation and the weather. All spectra were examined visually both before and after fitting the baseline, linear or polynomial. The collected spectra were transformed into IRAF data format using a modified FCRAO task 

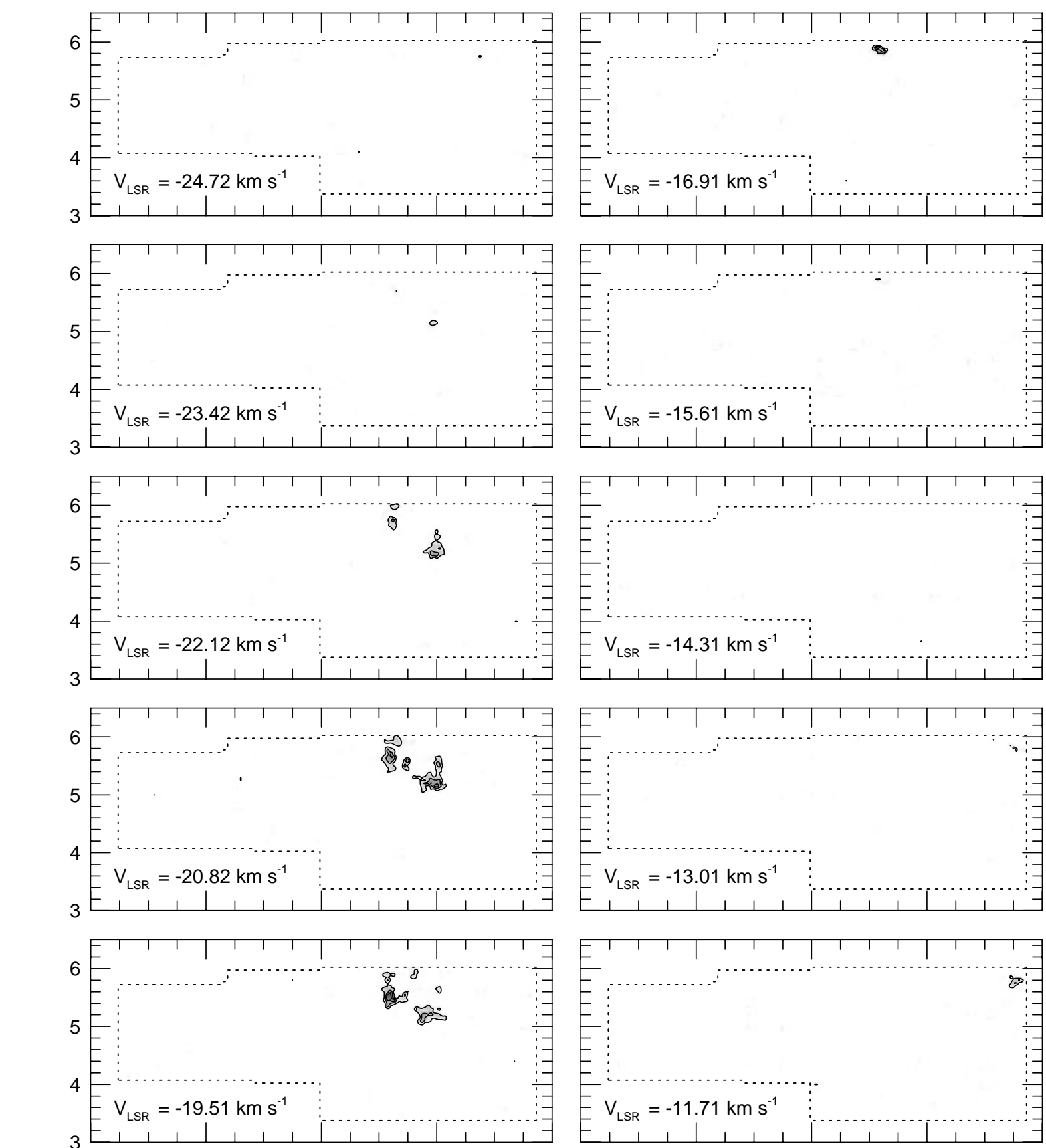

D
0
0
0
0
0
$\frac{1}{0}$
$\frac{0}{0}$
$\frac{0}{0}$
$\frac{0}{0}$
0
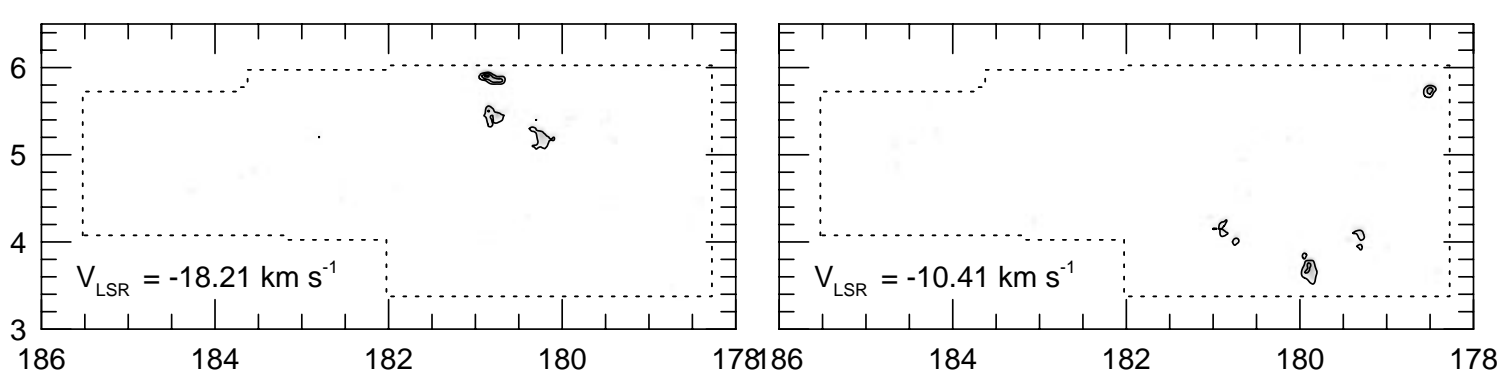

Galactic Longitude [deg]

Galactic Longitude [deg]

Fig. 3. ${ }^{12} \mathrm{CO}$ velocity maps of the Galactic Anticenter. Two velocity channels are binned together in each map; thus, each map has a velocity range of $1.3 \mathrm{~km} \mathrm{~s}^{-1}$. The grey scale range is 0.4 to $8 \mathrm{~K} \mathrm{~km} \mathrm{~s}^{-1}$. The lowest four contours are $1,2.5,4,6 \mathrm{~K} \mathrm{~km}$ $\mathrm{s}^{-1}$, and the increment between levels above $6 \mathrm{~K} \mathrm{~km} \mathrm{~s}^{-1}$ is $2 \mathrm{~K} \mathrm{~km} \mathrm{~s}^{-1}$. The dotted box represents the actual mapped area 

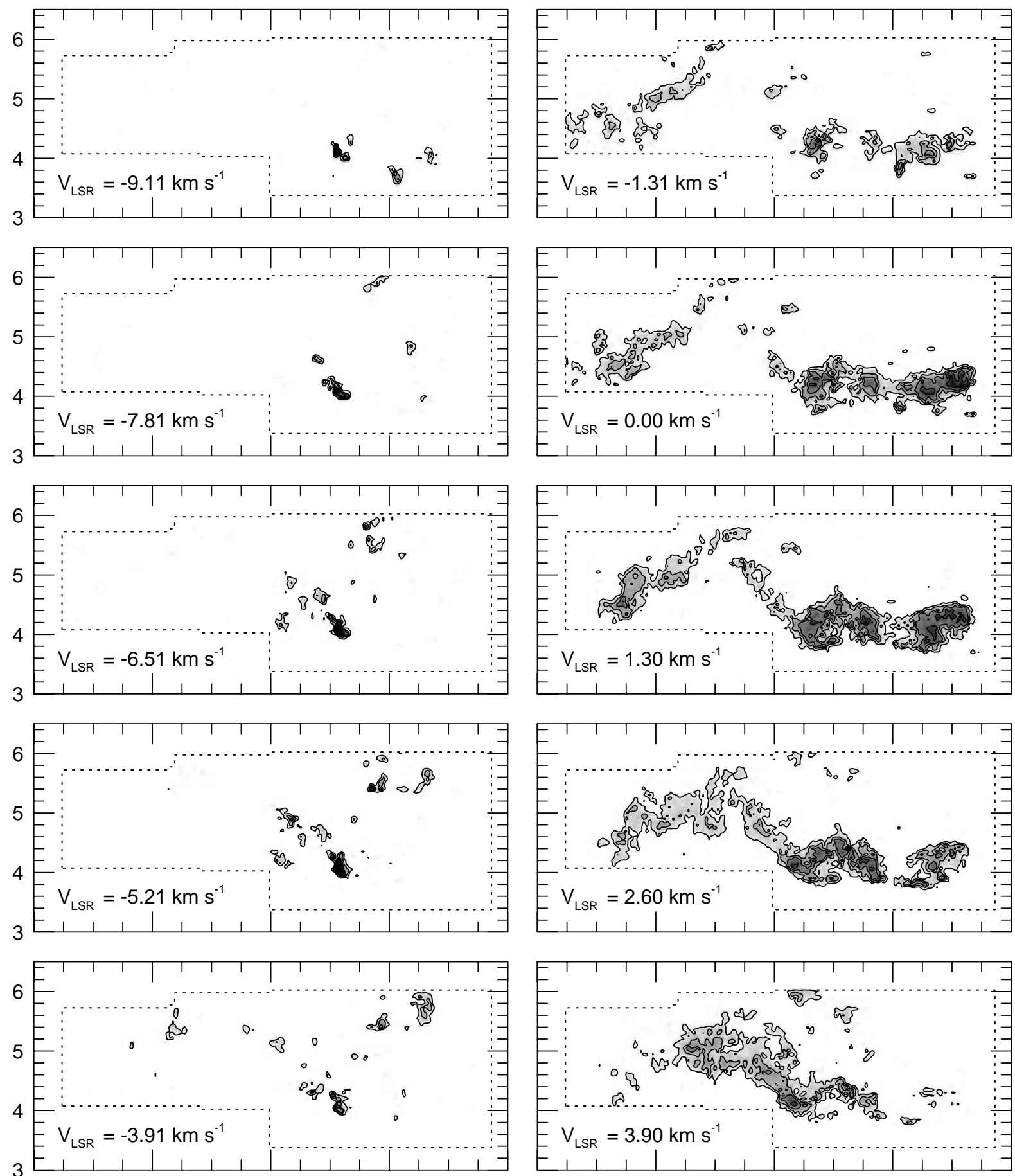

D্
$\frac{0}{0}$
0
$\frac{0}{0}$
$\frac{1}{0}$
$\frac{0}{0}$
$\frac{0}{0}$
$\frac{0}{0}$
0
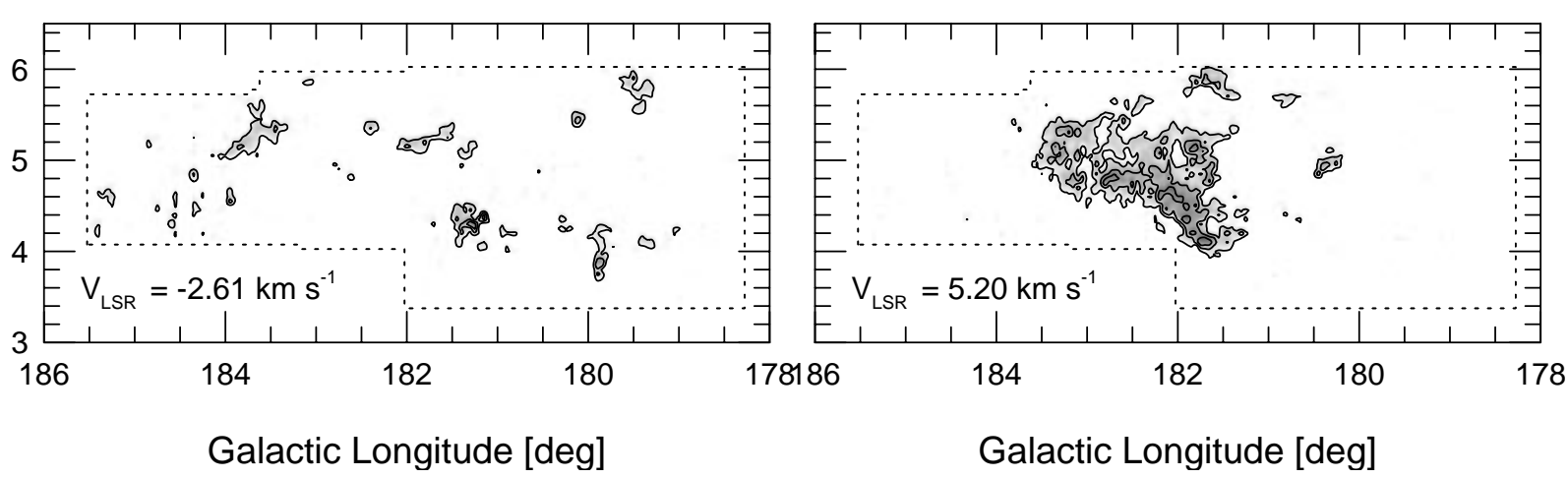

Fig. 3. continued

Galactic Longitude [deg] 

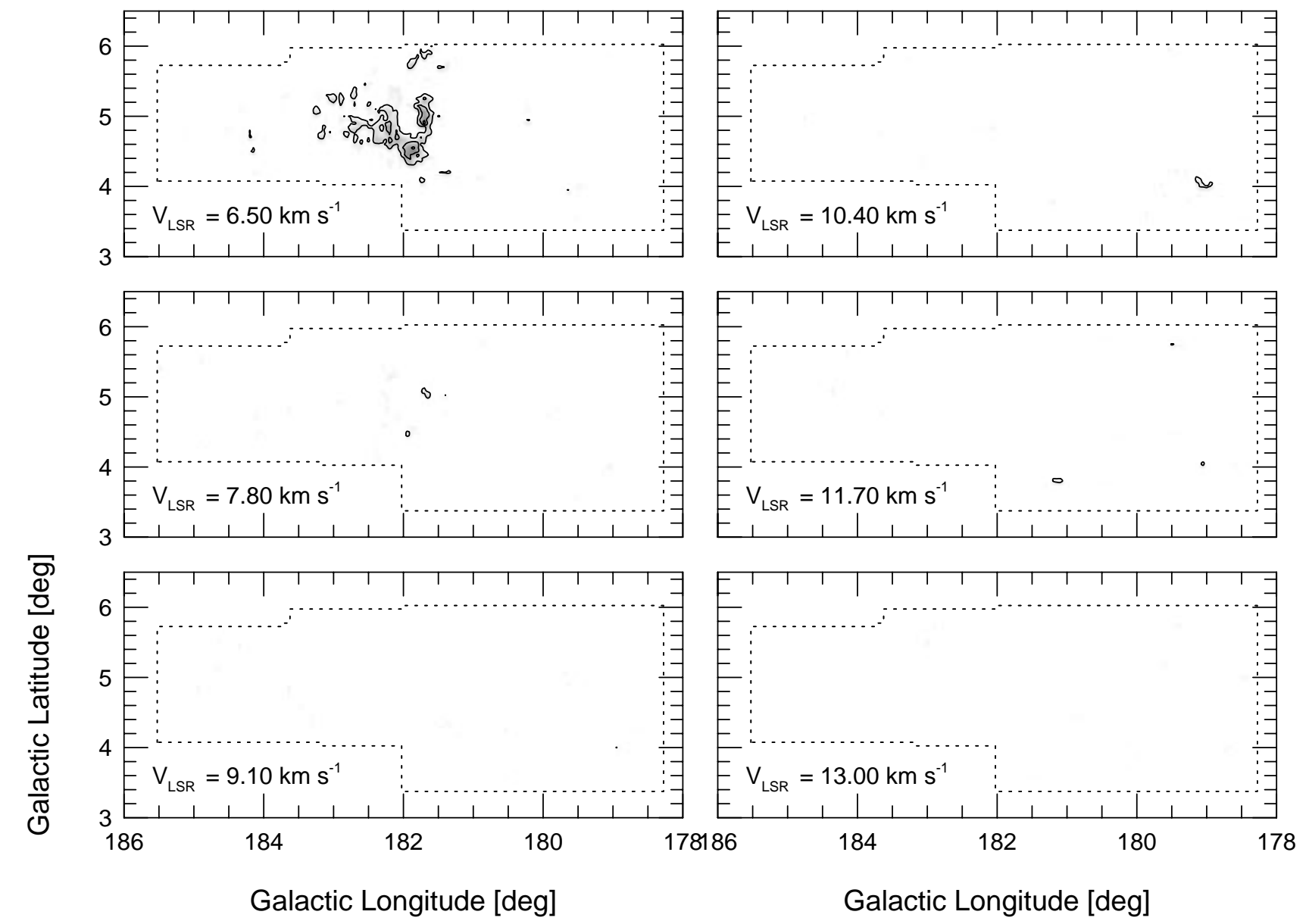

Fig. 3. continued

package developed at the Five College Radio Astronomy Observatory. Most of the subsequent analysis was done within IRAF using this package. The average rms noise level of the data is estimated to be $\Delta T_{\mathrm{rms}} \sim 0.25 \mathrm{~K}$ in $T_{\mathrm{R}}^{*}$ at a velocity resolution of $0.65 \mathrm{~km} \mathrm{~s}^{-1}$.

\section{Results}

A total number of about 7000 spectra has been obtained with a grid spacing of $3^{\prime}$ in the ${ }^{12} \mathrm{CO}(J=1-0)$ line. The mapped region covers most of the selected area (see Fig. 1), and is presented with dotted lines in Fig. 2. The total CO line intensity map, integrated from $v_{\mathrm{LSR}}=-25$ to $15 \mathrm{~km} \mathrm{~s}^{-1}$, which covers the whole emission range, is represented in Fig. 2. There are several warm clumps in the main emission region, some of which correspond to regions with strong dust emission. The overlayed filled circles in Fig. 2 are the IRAS point sources with $S_{60}>1 \mathrm{Jy}$ and $S_{100}>S_{60}$. Outside the main emission area, several small patches of molecular gas are also present. Overall, spatial coincidence and close morphological similarity is found between the $\mathrm{CO}$ emission and the FIR dust emission as in the case of an isolated cloud (see Fig. 1). The correlation between CO emission and FIR dust emission will be discussed in Sect. 6 .

We present our survey results in two standard ways: $l-b$ contour maps integrated over a range of velocity bins, and $v-b$ contour maps integrated over a range of longitude bins. Maps at individual velocities are shown in Fig. 3. The velocity coverage of each map is $1.3 \mathrm{~km} \mathrm{~s}^{-1}$, which corresponds to two channels of the $250 \mathrm{KHz}$ filterbank. Only $v_{\mathrm{LSR}}=-24.72 \mathrm{~km} \mathrm{~s}^{-1}$ to $+13.00 \mathrm{~km} \mathrm{~s}^{-1}$ is presented in this figure, as little emission is found in the rest of the velocity range. As in Fig. 2, the dotted box indicates the mapped area. The CO emission toward the Galactic Anticenter is generally weak, and the lowest contour of Fig. 3 is set to $1 \mathrm{~K} \mathrm{~km} \mathrm{~s}^{-1}(\sim 4 \sigma)$. In addition to contours, we employ grey-scale to discriminate the weaker emission regions from the stronger emission regions, which often appear in the typical survey data.

Figure 4 is a sequence of $b-v$ maps integrated for 0.25 along the longitude direction, corresponding to 5 pixels in our sampling. The lowest contour is set to $2.5 \mathrm{~K}(\sim 5 \sigma)$, and a grey-scale is employed to discriminate the weaker emission region as in the case of Fig. 3. These figures represent some obviously separate clouds overlapping along 

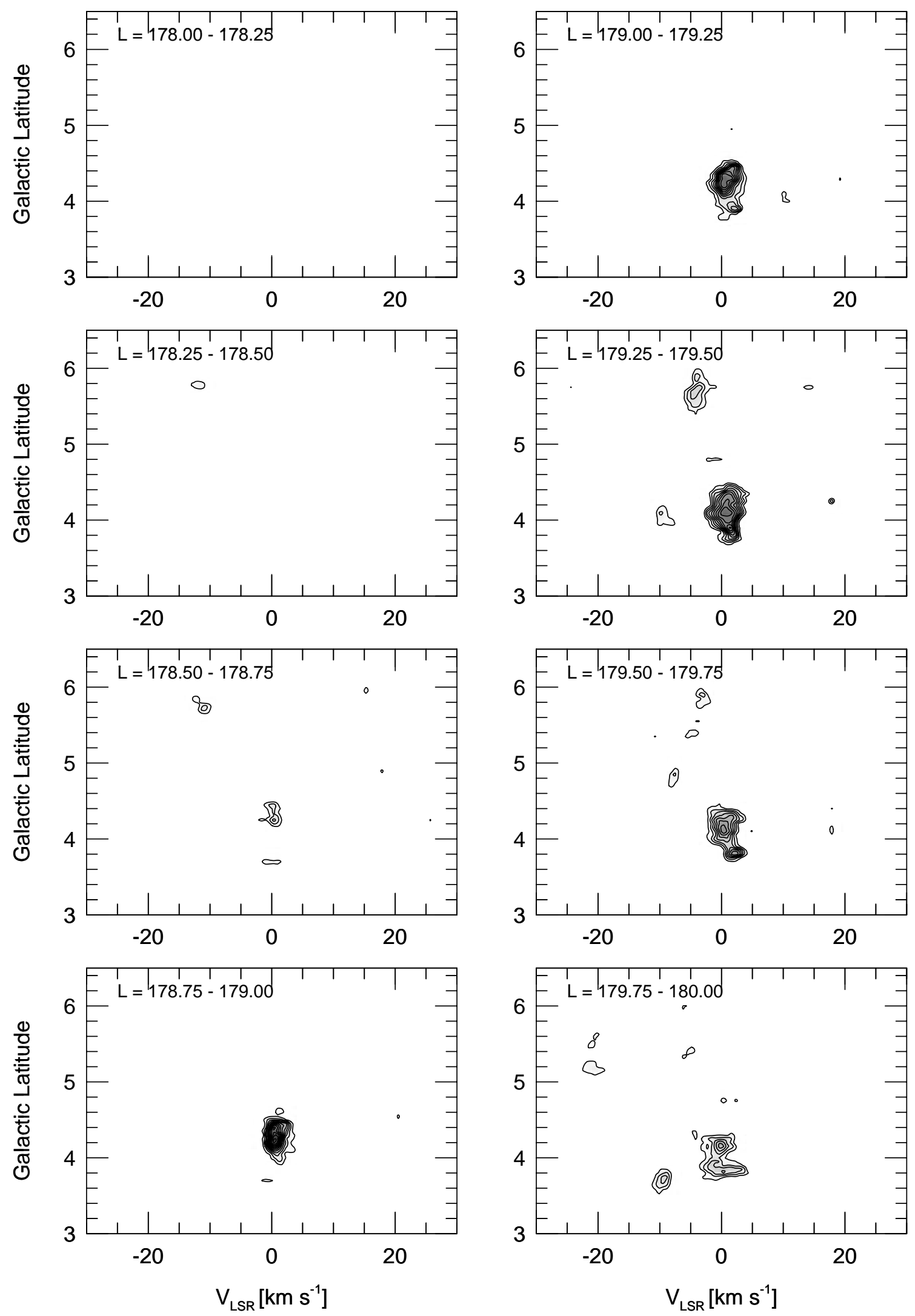

Fig. 4. Position-velocity maps (Galactic latitude-velocity). The grey scale range is 2.5 to $40 \mathrm{~K}$. The lowest contour level and the increment between levels are $2.5 \mathrm{~K}$ 

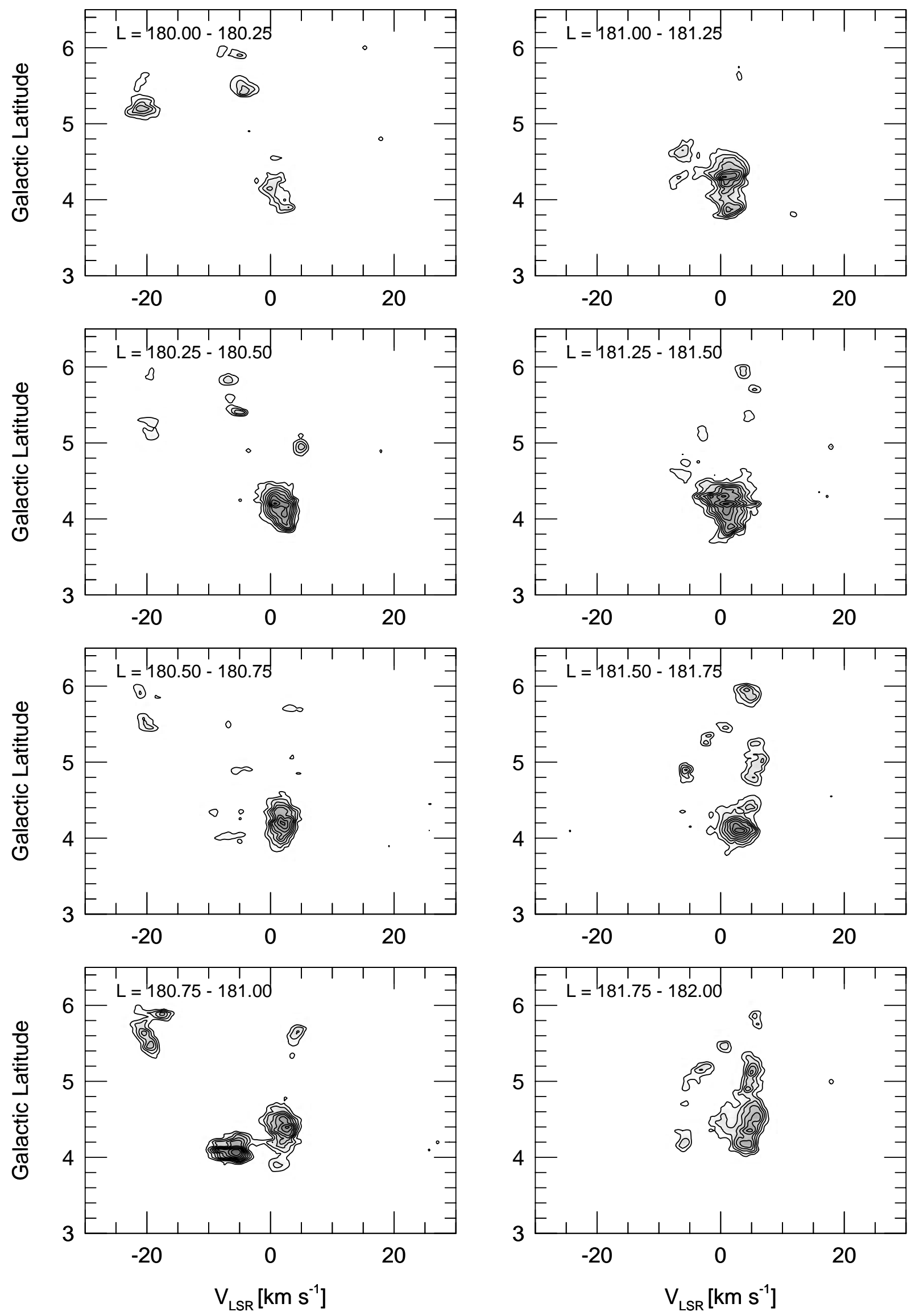

Fig. 4. continued 
Y. Lee et al.: Galactic anticenter CO survey. I.
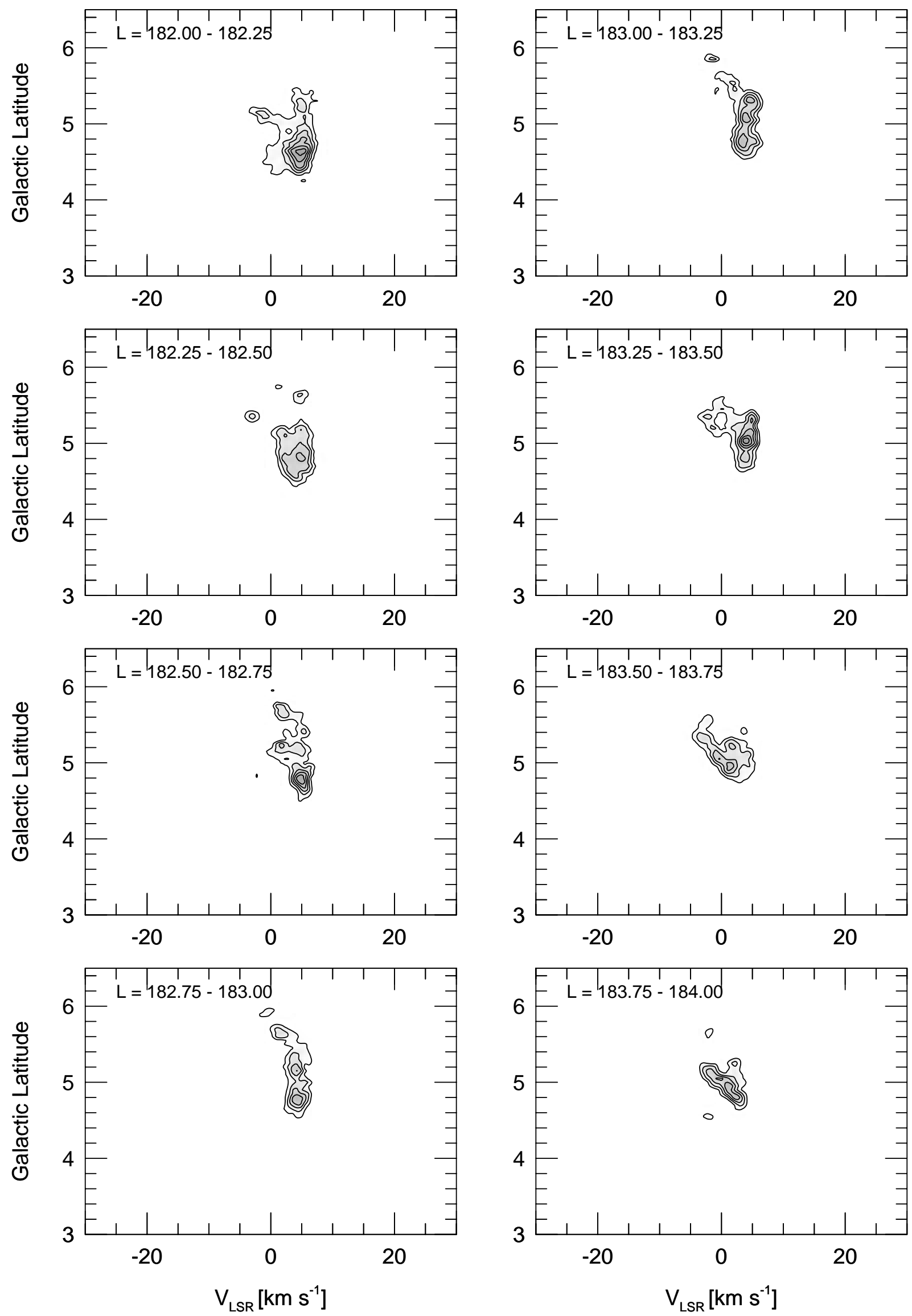

Fig. 4. continued 

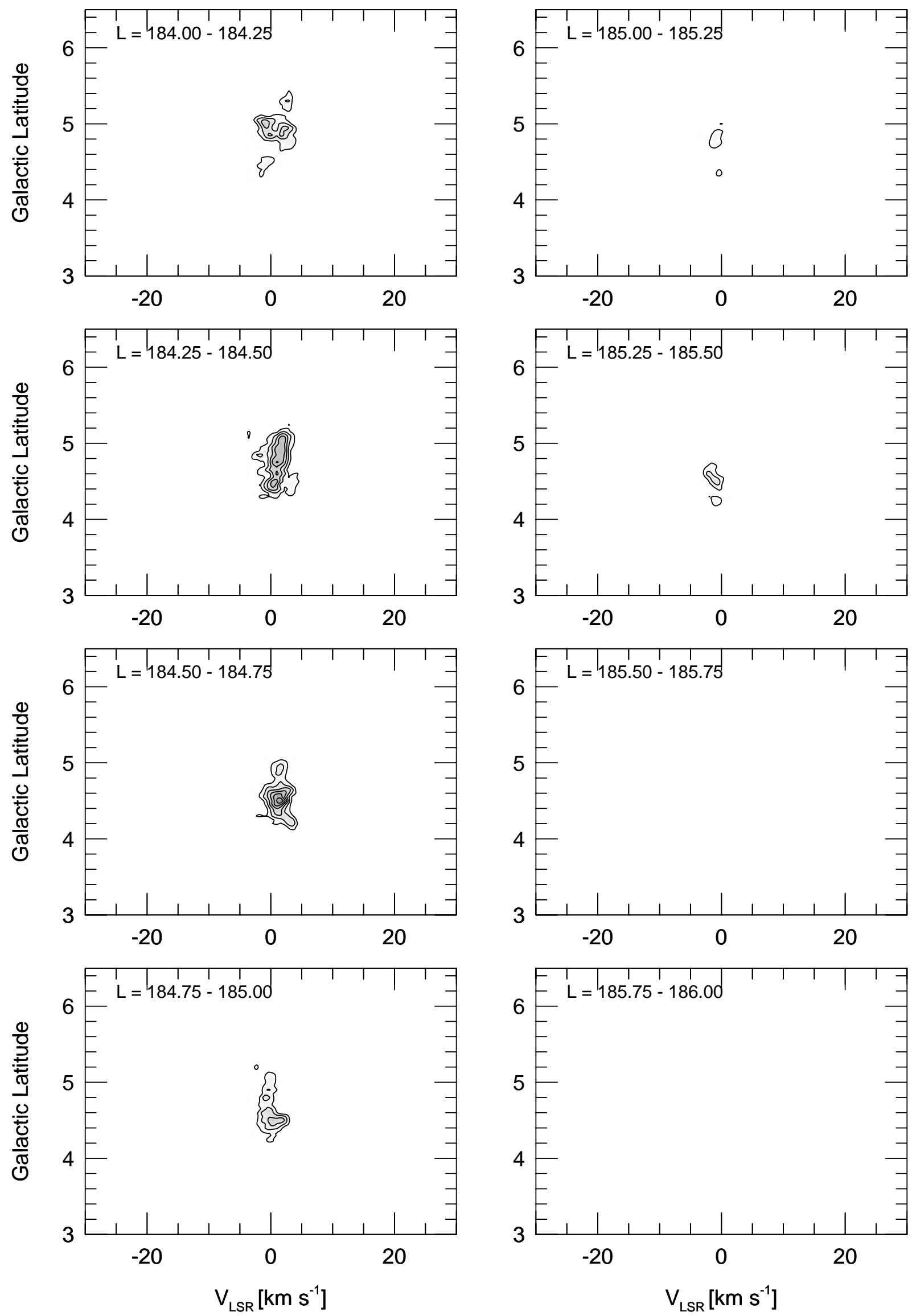

Fig. 4. continued 
the same lines of sight, which will be described in the following section.

\section{Cloud identification}

The major portion of the molecular gas is within a velocity range of -10 to $10 \mathrm{~km} \mathrm{~s}^{-1}$, and there are several pieces of small isolated clouds scattered on the small scale, especially in the region of $(l, b)=\left(178^{\circ} \sim 180^{\circ}, 5^{\circ} \sim 6^{\circ}\right)$ (see Figs. 3 and 4). However, it is also found that some portion of the $\mathrm{CO}$ emission shows more than one peak; many spectra show evidence that two or more clouds are blended together in some directions, mostly from $l=179^{\circ}$ to $182^{\circ}$ (see Sect. 5).

To effectively identify these clouds from the 3 dimensional data cube set $(l, b, v)$, we used a code developed by Lee et al. (1997), which is working as a user task within IRAF. Using the ${ }^{12} \mathrm{CO}$ cube data, they define a cloud to be an object composed of all pixels in longitude, latitude, and velocity that are simply connected and that lie above some threshold temperature. This is the same method employed by Scoville et al. (1987) and Lee et al. (1990). Ideally, one would like to define clouds with a $0 \mathrm{~K}$ threshold temperature. However, low threshold temperatures are impractical in view of the noise level in the spectra and more importantly because of the blending of adjacent clouds which occurs in the observed region. On the other hand, with too high a threshold temperature, regions are severely truncated, and it is impossible to obtain a reliable estimate of the sizes and thus the masses of the clouds. To define clouds we should choose a reasonable threshold temperature. Above the arbitrary threshold temperature, only those clouds with 2 or more pixels in each $l, b$, and $v$ are retained. In this way, we identify 30 clouds with a threshold temperature of $1.25 \mathrm{~K}(=5 \sigma)$. One of those is found to be very large, and seems to be blended with several adjacent clouds with similar velocities. Using a higher threshold temperature of $2.5 \mathrm{~K}(=10 \sigma)$ we have separated the object into several subclouds. The number of subclouds determined in this way is 12 , and the identified clouds and subclouds are listed in Table 1. Intriguingly, the subclouds appear in two distinct mean velocity groups, one with $v_{\mathrm{LSR}} \sim$ $1 \mathrm{~km} \mathrm{~s}^{-1}$, and the other with $v_{\mathrm{LSR}} \sim 4 \mathrm{~km} \mathrm{~s}^{-1}$, which also implies that the largest cloud (No. 18) is a blended one. The first column is the cloud number; centroid positions and their dispersions are given Cols. $2-5$. Column 6 is the centroid velocity and Col. 7 is the linewidth at half maximum. Peak temperature and the number of pixels retained within the cloud follow in Cols. 8 and 9. In this table we do not include detailed physical parameters, as distances are yet to be determined, though the distance of cloud No. 8 was found to be $4.7 \mathrm{kpc}$, which is associated with the HiI region Sh 241 (Moffat et al. 1979).

Recently, Kawamura et al. (1998) listed the clouds in this region using their ${ }^{13} \mathrm{CO}$ survey conducted with the

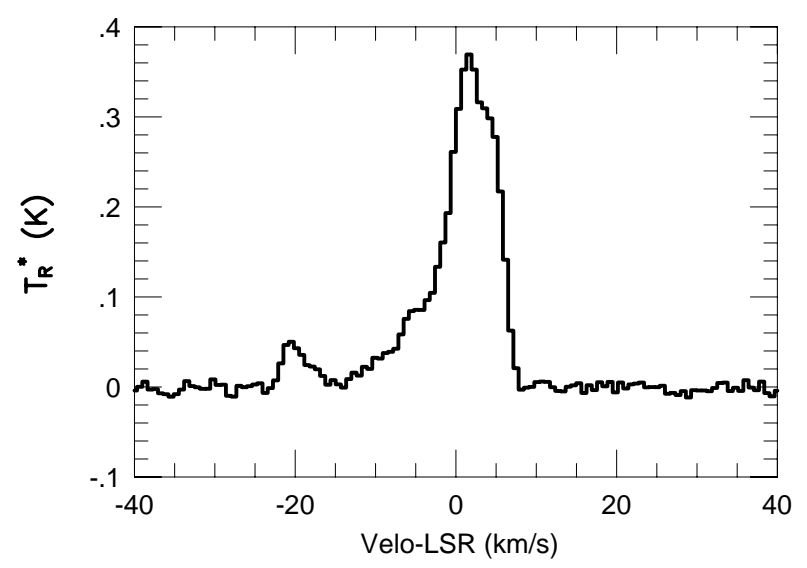

Fig. 5. Average of all 7000 spectra. While there is sharp cut in the positive-velocity emission regime at $7 \mathrm{~km} \mathrm{~s}^{-1}$, there is more extended emission in a negative-velocity regime up to $-25 \mathrm{~km} \mathrm{~s}^{-1}$

4-m telescope in Nagoya University. The number of clouds identified in their survey was 7 , much smaller than the number of clouds identified in this work. The Kawamura et al. (1998)'s distance determination toward these clouds $(d=1.1 \mathrm{kpc})$ was based on Racine (1968)'s photometry of a star $\mathrm{BD}+30^{\circ} 1096$. However, the coordinate of the star $(l, b)=\left(180^{\circ} .7,4.3\right)$ is very close to the HII region Sh 241 , the distance of which is known to be $4.7 \mathrm{kpc}$ (Moffat et al. 1979), which is by far more distant than those of the presumably local clouds. Thus, the distances of the clouds with noticeable opaqueness are likely ill-determined and should be redetermined. In fact, we are imaging these regions with deep CCD observations to estimate the distances, and for further analysis. The physical properties of the identified clouds will be presented in a separate paper with determination of distances of the clouds (Lee et al. in preparation).

\section{Some highlights}

Below we present some of the survey results and some discussion.

1. Most of the CO emission is confined within the velocity range from -10 to $10 \mathrm{~km} \mathrm{~s}^{-1}$. This can also be confirmed from Fig. 5, which is the average of the 7000 spectra comprising the entire region mapped. In addition, a couple of small clouds with anomalous velocities $\left(v_{\mathrm{LSR}}=-20 \mathrm{~km} \mathrm{~s}^{-1}\right)$ toward $l=180^{\circ}$ and $b=5^{\circ}$ to $6^{\circ}$ are identified. Their velocities of $-20 \mathrm{~km} \mathrm{~s}^{-1}$ are exceptional for this direction. Brand \& Blitz (1993) reported a streaming motion in the outer Galaxy in their study of its velocity field. Considering their estimate of a streaming motion of $3.8 \mathrm{~km} \mathrm{~s}^{-1}$ and possible local random motion, the velocity of $-20 \mathrm{~km} \mathrm{~s}^{-1}$ at the exact Antigalactic Center region is therefore quite exceptional. Five HiI regions (Sh $231-$ Sh 235) with abnormal velocities $\left(v_{\mathrm{LSR}}=-13\right.$ to $-23 \mathrm{~km} \mathrm{~s}^{-1}$ ) are found (Blitz et al. 1982; Brand \& Blitz 
Table 1. Identified clouds and subclouds

\begin{tabular}{|c|c|c|c|c|c|c|c|c|}
\hline No. & $\begin{array}{c}<l> \\
{[\text { degree] }}\end{array}$ & $\begin{array}{c}\sigma_{1} \\
{[\text { degree] }}\end{array}$ & $\begin{array}{c}\langle b> \\
{[\text { degree] }}\end{array}$ & $\begin{array}{c}\sigma_{\mathrm{b}} \\
{[\text { degree }]}\end{array}$ & $\begin{array}{c}<v_{\mathrm{LSR}}> \\
{\left[\mathrm{km} \mathrm{s}^{-1}\right]}\end{array}$ & $\begin{array}{c}\Delta v_{\mathrm{LSR}} \\
{\left[\mathrm{km} \mathrm{s}^{-1}\right]}\end{array}$ & $\begin{array}{l}T_{\mathrm{pk}} \\
{[\mathrm{K}]}\end{array}$ & $\overline{\overline{N N p}}$ \\
\hline 1 & 180.10 & 0.11 & 5.20 & 0.07 & -20.08 & 2.69 & 3.65 & 124 \\
\hline 2 & 180.81 & 0.05 & 5.64 & 0.17 & -18.93 & 3.37 & 5.40 & 120 \\
\hline 3 & 180.71 & 0.03 & 5.95 & 0.04 & -20.70 & 1.46 & 2.76 & 12 \\
\hline 4 & 179.97 & 0.02 & 5.53 & 0.07 & -20.39 & 1.65 & 2.28 & 23 \\
\hline 5 & 180.53 & 0.03 & 5.54 & 0.05 & -19.81 & 1.86 & 2.86 & 15 \\
\hline 6 & 178.48 & 0.05 & 5.75 & 0.04 & -10.71 & 1.70 & 3.50 & 16 \\
\hline 7 & 179.88 & 0.04 & 3.68 & 0.06 & -9.18 & 1.89 & 4.13 & 32 \\
\hline 8 & 180.85 & 0.07 & 4.10 & 0.09 & -5.76 & 3.92 & 13.18 & 222 \\
\hline 9 & 179.30 & 0.01 & 4.08 & 0.04 & -9.16 & 1.60 & 3.18 & 10 \\
\hline 10 & 181.16 & 0.06 & 4.64 & 0.06 & -6.02 & 2.22 & 3.14 & 42 \\
\hline 11 & 180.38 & 0.02 & 5.82 & 0.02 & -6.07 & 1.42 & 3.67 & 12 \\
\hline 12 & 181.82 & 0.05 & 4.18 & 0.05 & -5.52 & 1.35 & 2.93 & 25 \\
\hline 13 & 181.46 & 0.02 & 4.55 & 0.06 & -5.42 & 1.49 & 2.96 & 12 \\
\hline 14 & 180.18 & 0.08 & 5.45 & 0.06 & -4.09 & 2.62 & 5.67 & 68 \\
\hline 15 & 181.67 & 0.05 & 4.90 & 0.06 & -5.10 & 1.63 & 4.27 & 31 \\
\hline 16 & 179.38 & 0.06 & 5.67 & 0.10 & -3.45 & 2.03 & 3.08 & 87 \\
\hline 17 & 181.96 & 0.10 & 5.14 & 0.05 & -1.72 & 2.86 & 2.52 & 56 \\
\hline 18 & 181.36 & 1.63 & 4.43 & 0.39 & 1.58 & 4.30 & 8.31 & 7148 \\
\hline $\mathrm{a}$ & 181.14 & 0.53 & 4.24 & 0.18 & 1.57 & 3.75 & 8.31 & 1232 \\
\hline b & 179.89 & 0.04 & 3.84 & 0.04 & -0.40 & 1.70 & 5.13 & 28 \\
\hline $\mathrm{c}$ & 179.21 & 0.26 & 4.18 & 0.16 & 0.45 & 1.75 & 7.58 & 736 \\
\hline $\mathrm{d}$ & 184.52 & 0.08 & 4.51 & 0.10 & 0.45 & 1.51 & 4.66 & 73 \\
\hline $\mathrm{e}$ & 184.35 & 0.06 & 4.77 & 0.04 & 0.40 & 1.16 & 3.11 & 12 \\
\hline $\mathrm{f}$ & 181.04 & 0.05 & 3.90 & 0.03 & 1.06 & 2.10 & 4.10 & 18 \\
\hline $\mathrm{g}$ & 184.44 & 0.05 & 5.00 & 0.06 & 1.46 & 1.18 & 3.34 & 17 \\
\hline $\mathrm{h}$ & 183.34 & 0.06 & 5.05 & 0.05 & 3.70 & 1.39 & 4.26 & 40 \\
\hline $\mathrm{i}$ & 183.12 & 0.04 & 4.81 & 0.05 & 3.15 & 1.58 & 3.15 & 12 \\
\hline $\mathrm{j}$ & 182.74 & 0.08 & 4.78 & 0.04 & 4.13 & 1.37 & 3.93 & 34 \\
\hline $\mathrm{k}$ & 182.23 & 0.03 & 4.87 & 0.04 & 5.31 & 1.82 & 3.10 & 10 \\
\hline 1 & 181.77 & 0.07 & 5.04 & 0.09 & 4.72 & 2.10 & 4.05 & 39 \\
\hline 19 & 179.52 & 0.04 & 5.89 & 0.05 & -2.63 & 1.77 & 3.08 & 18 \\
\hline 20 & 182.40 & 0.03 & 5.36 & 0.03 & -2.30 & 1.09 & 2.15 & 13 \\
\hline 21 & 185.33 & 0.07 & 4.52 & 0.05 & -0.66 & 1.82 & 2.81 & 21 \\
\hline 22 & 184.96 & 0.05 & 4.86 & 0.10 & -0.05 & 0.50 & 2.97 & 42 \\
\hline 23 & 181.94 & 0.05 & 4.51 & 0.05 & -0.04 & 0.94 & 2.89 & 38 \\
\hline 24 & 181.75 & 0.08 & 5.47 & 0.03 & 0.11 & 0.76 & 2.76 & 31 \\
\hline 25 & 182.80 & 0.03 & 5.64 & 0.04 & 1.03 & 1.94 & 2.65 & 22 \\
\hline 26 & 182.58 & 0.05 & 5.68 & 0.05 & 1.52 & 1.75 & 2.05 & 29 \\
\hline 27 & 181.61 & 0.10 & 5.90 & 0.06 & 3.95 & 2.27 & 3.37 & 88 \\
\hline 28 & 180.79 & 0.05 & 5.61 & 0.06 & 3.66 & 1.46 & 2.64 & 31 \\
\hline 29 & 182.40 & 0.05 & 5.61 & 0.05 & 4.22 & 0.99 & 2.45 & 15 \\
\hline 30 & 180.36 & 0.07 & 4.93 & 0.05 & 4.29 & 1.06 & 2.90 & 24 \\
\hline
\end{tabular}

1993) around $l=173^{\circ}$. Heyer et al. (1996) observed these objects in ${ }^{12} \mathrm{CO}$ and ${ }^{13} \mathrm{CO}$, but no specific discussion on the velocities on these objects was included. More detailed study on this abnormal velocity field may clarify the physical and dynamical status of the clouds within it. In fact, we are observing these objects at higher resolution in several molecular transitions to analyze the physical status of the clouds. Figure 6 shows a spatial-velocity map integrated along the latitude of 3.5 to 6 . 0 . This figure provides a longitudinal view of the velocity distribution, and also confirms that the $\mathrm{CO}$ emission is concentrated into the velocity range mentioned above. Some of the regions within this velocity range are likely to be nearby dark clouds, as they are clearly associated with noticeable opaque areas on the Palomar Observatory Sky Survey (POSS) plates. As mentioned previously, there are several Lynds clouds, which are generally local clouds.

2. The highest antenna temperature $\left(T_{\mathrm{R}}^{*}=13.2 \mathrm{~K}\right)$ is found at the position of $(l, b)=(180.8,4.0)$. This is close to the direction of the HiI region Sh 241, an active star forming region. The peak temperature map is not shown in this paper as it is similar to Fig. 2. Most of the clouds 


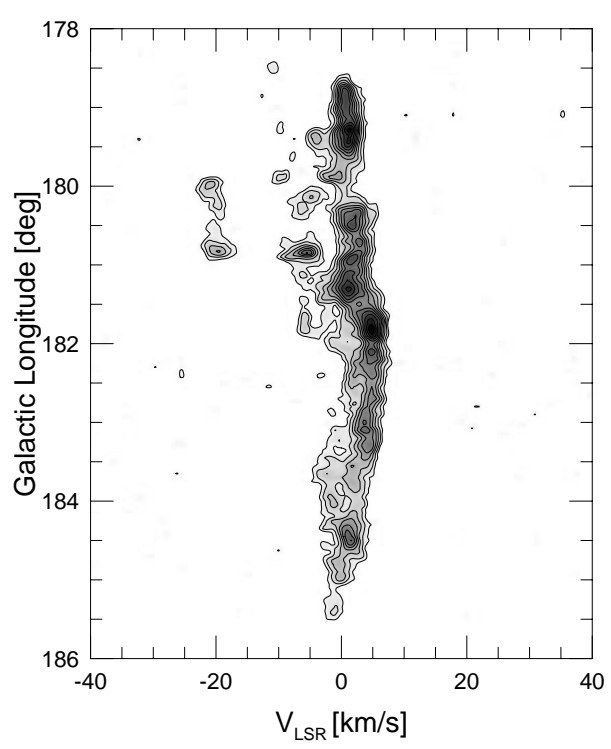

Fig. 6. Global integrated position-velocity map. The lowest contour is $4.5,9$, and $15 \mathrm{~K}$, and the increment between the levels above $15 \mathrm{~K}$ is $6 \mathrm{~K}$. The grey scale range is 2 to $60 \mathrm{~K}$

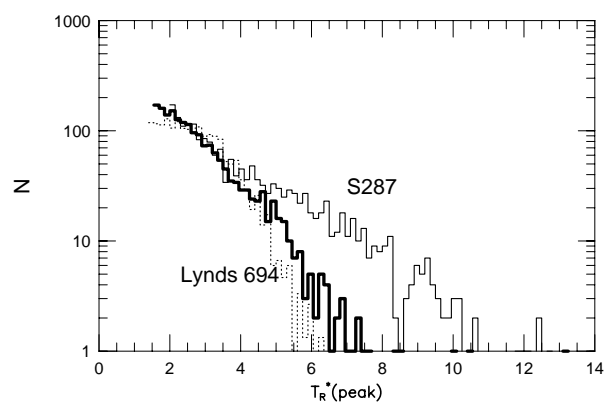

Fig. 7. The peak ${ }^{12} \mathrm{CO}$ temperature distribution of three different clouds; Lynds 694 (dotted line), Sh 287 (solid line), and the mapped region in this study (thick solid line)

have $T_{\mathrm{R}}^{*}<6 \mathrm{~K}$ and the average $T_{\mathrm{R}}^{*}$ is about $3.5 \mathrm{~K}$. This is illustrated in the histogram (Fig. 7). In this figure the $\mathrm{CO}$ peak temperature distributions of three molecular gas regions are presented; a typical GMC associated with the HII region Sh 287 (Lee 1994), a typical dark cloud, Lynds 694 (Lee \& Lee in preparation) without star forming activity, and the region mapped in this work. Only those pixels above the $5 \sigma$ level are entered for the target region (1 800 spectra), and the numbers of pixels of Sh 287 and Lynds 694 are normalized to that of our target region mapped. Clearly, CO emission of the Galactic Anticenter molecular gas is similar to that of Lynds 694, a dark cloud in the solar neighborhood, and is substantially weaker than that of the GMCs with some exceptional portions directly associated with Sh 241. Thus, most of the region may have similar properties to those of dark clouds in the solar neighborhood, though this remains to be determined in future work.

3. Several CO emission peaks arise around IRAS point sources (see Fig. 2), some of which are associated with

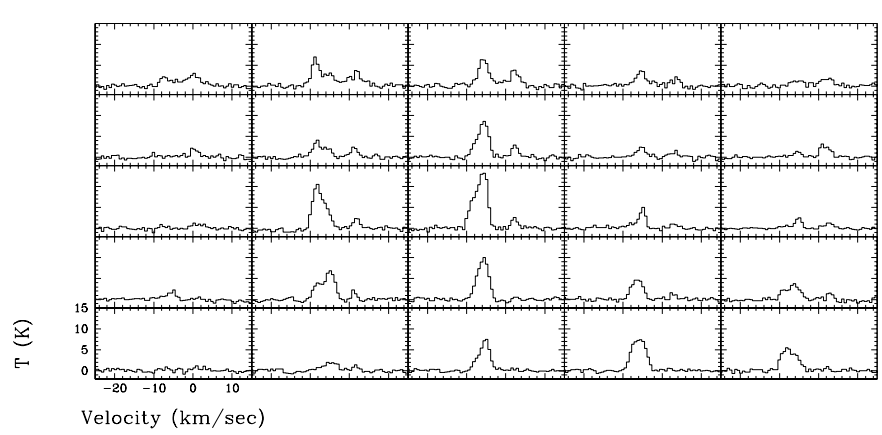

Fig. 8. Example of spectra at the position of $(l, b)=$ $\left(180^{\circ} 85,4.10\right)$ shows a possible bipolar outflow feature

HII regions and possible bipolar outflows, implying that star forming activity is clearly going on. At the position $(l, b)=\left(180^{\circ} 85,4^{\circ} .10\right)$ there is a feature similar to bipolar outflow (Fig. 8), though this should be confirmed with higher resolution observations. Only those IRAS point sources with conspicuous flux $\left(S_{60}>1 \mathrm{Jy}\right)$ are shown in Fig. 2; the weaker point sources which are prevalent all over the $\mathrm{CO}$ emission boundary are excluded. It is notable that IRAS point sources with conspicuous flux are all confined to the region within $l<182^{\circ}$, which has stronger CO emission than the rest of the region. In fact, the average temperature of the remaining portion $\left(l>182^{\circ}\right)$ is at least $2 \mathrm{~K}$ lower, which is strongly implying that there exists a substantial amount of colder gas features without internal heating sources, as well as brighter and warmer components associated with internal heating sources. However, the $\mathrm{CO}$ emission peaks are not as bright as those of local GMCs containing HII regions. This may be caused in part by beam dilution effects. In fact, the distance of the HII region Sh 241 was estimated as $4.7 \mathrm{kpc}$ (Moffat et al. 1979). The case for beam dilution is also supported by the fact that the brightest lines in each peak are very localized. According to Moffat et al. (1979), the central star of Sh 241 is an O9 star, however, on the wisp of nebulosity there is another OB star, the distance of which is over $10 \mathrm{kpc}$ from the sun, though this needs to be confirmed. If their estimate was correct, then it would confirm star forming activity at one of the most distant regions in the outer Galaxy.

4. The linewidths of the $\mathrm{CO}$ emission of the clouds $\left(\Delta v=1\right.$ to $4 \mathrm{~km} \mathrm{~s}^{-1}$; see Table 1) are generally narrower than those of local GMCs $\left(\Delta v \geq 5 \mathrm{~km} \mathrm{~s}^{-1}\right.$; Blitz 1987), and more or less similar to those of dark clouds in the solar neighborhood. The peak temperature ranges from 2 to $8 \mathrm{~K}$ except for one cloud directly associated with Sh 241. This implies that the most of the CO emission comes from cold dark clouds, which normally do not have large bulk motions. 


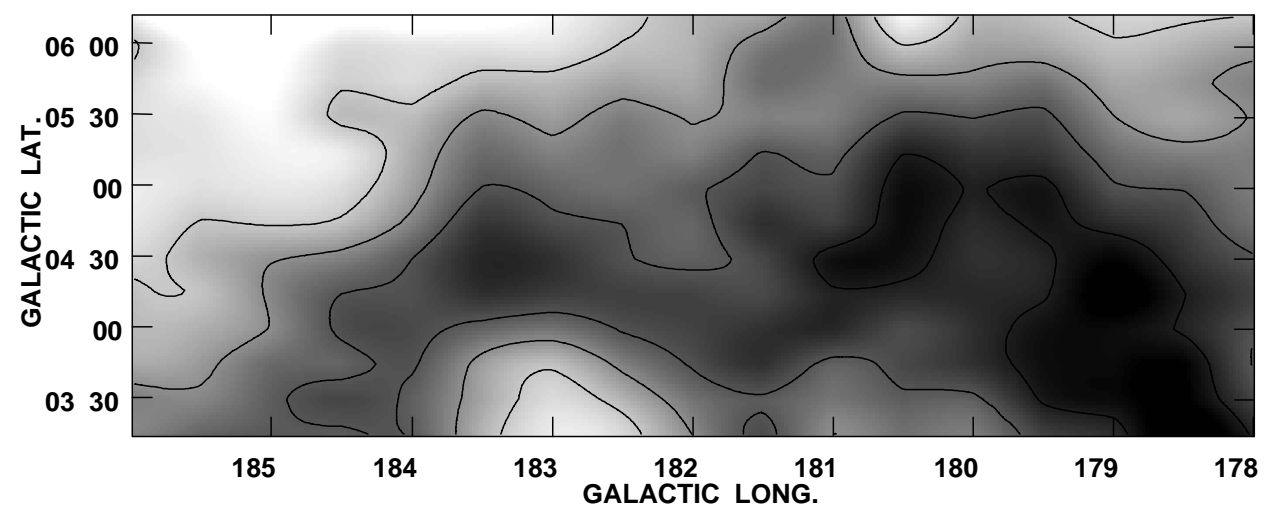

Fig. 9. HI integrated intensity map. The lowest contour is $1700 \mathrm{~K} \mathrm{~km} \mathrm{~s}^{-1}$, and the increment between the contours is $100 \mathrm{~K}$ $\mathrm{km} \mathrm{s}^{-1}$. The grey scale range is 1600 to $2000 \mathrm{~K} \mathrm{~km} \mathrm{~s}^{-1}$

\section{Correlation with dust emission}

Both FIR continuum emission from dust and CO spectral line emission can be used to estimate the masses of interstellar clouds, and the FIR data can be compared for consistency and/or calibration with the CO data. For isolated clouds, spatial coincidence and close morphological similarity is found between the $\mathrm{CO}$ emission and dust emission, especially after subtraction of the extended background Galactic FIR emission (Heyer et al. 1987; Langer et al. 1989; and Mooney 1992). In general, an accurate separation of the background Galactic FIR emission from the cloud emission is necessary before using the IRAS data to estimate the FIR luminosity of a cloud. However, for the region in this study the identification of the FIR emission is fairly straightforward as the cloud is well isolated. The only other contribution to the FIR emission would be atomic hydrogen. An Hi map was available from the Weaver \& Williams (1973) data base, although the resolution of the original data is very large, $36^{\prime}$. We have resampled it onto a $3^{\prime}$ grid, assuming there is no specific structure. In Fig. 9 we present the Hi integrated intensity map fitted to the CO mapped region. The distribution of Hi emission is found to have a very small dynamic range of a factor of 1.3, whereas the CO emission without internal heating sources has a much larger dynamic range. Thus the atomic hydrogen does not affect significantly the slope of the relationship between the FIR emission and the $\mathrm{CO}$ emission; we have not included the HI contribution in this work.

Figure 10 represents the pixel to pixel correlation between the CO integrated intensity and dust FIR intensity at $100 \mu \mathrm{m}$. It shows two distinctly different features; a very scattered distribution of FIR intensity above $35 \mathrm{MJy}$ $\mathrm{sr}^{-1}$, and the bulk of emission confined to between 15 and $30 \mathrm{MJy} \mathrm{sr}^{-1}$. However, we note that the pixels with FIR intensity greater than $35 \mathrm{MJy} \mathrm{sr}^{-1}$ are all associated with the HII region Sh 241 and IRAS point sources. Except for those points, a good correlation is found between the FIR emission and the ${ }^{12} \mathrm{CO}$ integrated intensity. To

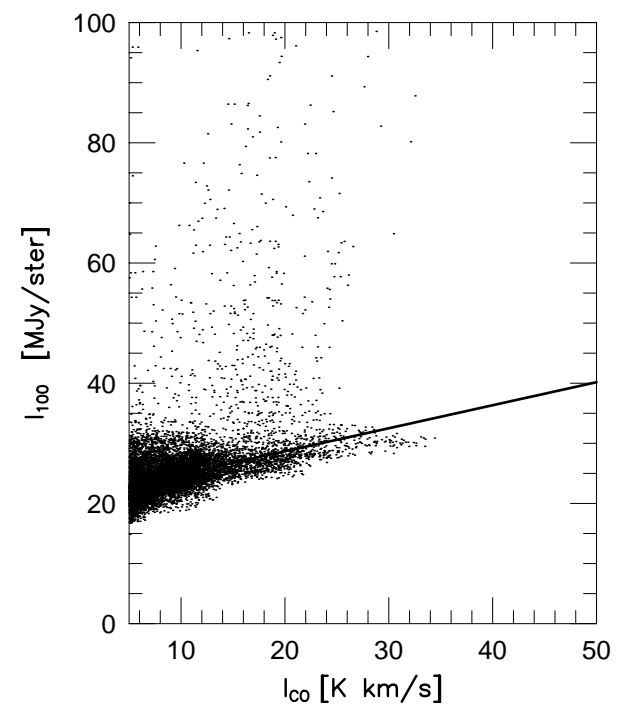

Fig. 10. The $100 \mu \mathrm{m}$ intensity as a function of CO integrated intensity. Points with $I_{100}$ less than $35 \mathrm{MJy} \mathrm{sr}^{-1}$ were fitted using the least-squares technique and the fitted line is shown. The points that lie substantially above the fitted line larger than $35 \mathrm{MJy} \mathrm{sr}^{-1}$ are due to IRAS point sources and Sh 241

determine the relation between ${ }^{12} \mathrm{CO}$ integrated intensity and FIR intensity, pixels with the CO integrated intensity $>5 \mathrm{~K} \mathrm{~km} \mathrm{~s}^{-1}(3 \sigma)$ have been fit by using the least-squares method. The slope of the correlation is $0.38\left(\mathrm{MJy} \mathrm{sr}^{-1}\right)$ $\left(\mathrm{K} \mathrm{km} \mathrm{s}^{-1}\right)^{-1}$ and the intercept is $21.0 \mathrm{MJy} \mathrm{sr}^{-1}$, which is represented as a thick solid line in Fig. 10. The nonzero intercept most likely represents emission from dust in a region which lacks ${ }^{12} \mathrm{CO}$. The amount of dust that lies in front of or behind the target region may not be small, as the intercept of above equation is nonnegligible. HI contribution to the FIR emission is also involved as mentioned previously. However, considering that it is the general dust emission from the Galaxy, thus not associated directly with the $\mathrm{CO}$ integrated intensity, only the slope represents the true relation between the ${ }^{12} \mathrm{CO}$ integrated intensity and the FIR intensity. The good correlation 
implies that there is little stray dust emission in the line of sight.

Numerous studies have found a linear relationship between $100 \mu \mathrm{m}$ intensity and either the HI column density in regions of atomic gas or the CO column density in molecular clouds (Boulanger \& Pérault 1988; Snell et al. 1989; Laureijs et al. 1995; Boulanger et al. 1998). Boulanger \& Pérault (1988) found ratios between the $100 \mu \mathrm{m}$ intensity and CO integrated intensity in the range 0.6 to $2.5\left(\mathrm{MJy} \mathrm{sr}^{-1}\right)\left(\mathrm{K} \mathrm{km} \mathrm{s}^{-1}\right)^{-1}$ with an average value of $1.4\left(\mathrm{MJy} \mathrm{sr}^{-1}\right)\left(\mathrm{K} \mathrm{km} \mathrm{s}^{-1}\right)^{-1}$ for regions outside of star forming sites. This value can be compared with the average slope found for the target region of 0.38 (MJy $\left.\mathrm{sr}^{-1}\right)\left(\mathrm{K} \mathrm{km} \mathrm{s}^{-1}\right)^{-1}$, substantially less than this value. Based on the slope of the least-squares fit to the data, we have computed the ratio of $100 \mu \mathrm{m}$ intensity to total hydrogen column density. We can use the CO conversion factor computed from the $\gamma$-ray analysis (Bloemen 1989), of $2.310^{20} \mathrm{H}_{2} \mathrm{~cm}^{-2}\left(\mathrm{~K} \mathrm{~km} \mathrm{~s}^{-1}\right)^{-1}$ to convert the $\mathrm{CO}$ intensities to hydrogen column densities. Expressing this ratio in terms of the hydrogen, the values found for the target region are $I_{100 \mu \mathrm{m}} / N(\mathrm{H})=0.07\left(\mathrm{MJy} \mathrm{sr}^{-1}\right)$ $\left(10^{20} \mathrm{H} \mathrm{cm}^{-2}\right)^{-1}$. These values can be compared to those for dark clouds; $I_{100 \mu \mathrm{m}} / N(\mathrm{H})$ is $0.07\left(\mathrm{MJy} \mathrm{sr}^{-1}\right)\left(10^{20} \mathrm{H}\right.$ $\left.\mathrm{cm}^{-2}\right)^{-1}$ ) for B18, and $0.10\left(\mathrm{MJy} \mathrm{sr}^{-1}\right)\left(10^{20} \mathrm{H} \mathrm{cm}^{-2}\right)^{-1}$ for HCL2 (Snell et al. 1989). The values for warmer GMCs are substantially higher; for example, in Orion Molecular Cloud (OMC): $I_{100 \mu \mathrm{m}} / N(\mathrm{H})=1.3\left(\mathrm{MJy} \mathrm{sr}^{-1}\right) /\left(10^{20}\right.$ $\left.\mathrm{H} \mathrm{cm}^{-2}\right)^{-1}$ (Boulanger \& Pérault 1988). Thus, the ratios found for the target region are comparable to the dark cloud ratios, and a factor of $\sim 20$ times smaller than the ratios in OMC. Snell et al. (1989) attributed the low ratios in the dark clouds to dust heated exclusively by the solar neighborhood interstellar radiation field. A similar conclusion was reached by Mooney (1992) for the clouds classified as IR-quiet. Thus, the low ratios found for the target region probably results from the absence of internal or significant nearby external heating sources. If this is the case, the dust temperature should also be relatively low as is seen in the dark clouds.

\section{Summary}

We have observed a 17 square-degree section $(l, b)=$ $\left(178^{\circ} .0 \sim 186^{\circ} .0,3.5 \sim 6.0\right)$ of the Galactic Anticenter region in ${ }^{12} \mathrm{CO} J=1-0$ using the 3 -mm SIS receiver on the 14-m telescope at Taeduk Radio Astronomy Observatory. A total of 7000 spectra has been obtained with a grid spacing of $3^{\prime}$. The results of the observations are presented in the form of $l-b$ and $v-b$ contour maps. Molecular emission toward the Galactic Anticenter region is found to be very extended, and matches well with the FIR emission boundary. The radial velocity of the molecular gas is found to be within the range of $v_{\mathrm{LSR}}=-25 \sim+10 \mathrm{~km} \mathrm{~s}^{-1}$. We have also found several small clouds located at $l=180^{\circ}$, $b=5^{\circ} \sim 6^{\circ}$ have $v_{\mathrm{LSR}}=-20 \mathrm{~km} \mathrm{~s}^{-1}$, which is quite anomalous in this direction. We have identified 30 individual clouds of the mapped region with an arbitrary threshold temperature using a cloud identification code within IRAF. Twelve subclouds are also identified with a higher threshold temperature from the largest cloud. There are several heating sources within the boundary of the region mapped. However, these are very localized, and do not seem to affect the surrounding dust on a large scale. Thus the bulk of the region lacks internal heating sources, and remains unheated. The primary heating source seems to be the general interstellar radiation field. The $\mathrm{CO}$ emission is well correlated with the FIR emission at $100 \mu \mathrm{m}$, which implies that there is little stray dust emission in the line of sight. The FIR emission and the ${ }^{12} \mathrm{CO}$ integrated intensity relationship finds that the highest-possible value of $I_{100} / I_{\mathrm{CO}}$ is $\sim 0.38\left(\mathrm{MJy} \mathrm{sr}^{-1}\right)\left(\mathrm{K} \mathrm{km} \mathrm{s}^{-1}\right)^{-1}$. This value is very close to that of normal dark clouds, thus the ratio of a substantial part of the mapped region is smaller than that of the dark clouds in the solar neighborhood.

Acknowledgements. Y.L. acknowledges the support for this work by Creative Research Fund 97-5400-003 of the Ministry of Science and Technology, Korea. We thanks to L. Allen and E. Bergin for their careful reading of the manuscript. We are also grateful to the referee, Dr. W.B. Burton, for his critical comments and kind suggestions.

\section{References}

Blitz L., 1987, in "Physical processes in interstellar clouds", Proceedings of the NATO Advanced Study Institute, Irsee. Dordrecht, D. Reidel Publishing Co., pp. 35-58

Blitz L., Fich M., Stark A.A., 1982, ApJS 49, 183

Bloemen J.B.G.M., 1989, ARA\&A 27, 469

Boulanger F., Pérault M., 1988, ApJ 330, 964

Boulanger F., Bronfman L., Dame T.M., Thaddeus P., 1998, A\&A 332, 273

Brand J., Blitz L., 1993, A\&A 275, 67

Brand J., Wouterloot J.G.A., 1994, A\&AS 103, 503

Brand J., Wouterloot J.G.A., 1995, A\&A 303, 851

Carpenter J.M., Snell R.L., Schloerb F.P., 1995, ApJ 445, 246

Combes F., 1991, ARA\&A 29, 195

Dame T.M., Ungerechts H., Cohen R.S., et al., 1987, ApJ 322, 706

de Geus E.J., Vogel S.N., Digel S.W., Gruendl R.A., 1993, ApJ 413, L97

Digel S., Thaddeus P., Bally J., 1990, ApJ 357, L29

Digel S., de Geus E., Thaddeus P., 1994, ApJ 422, 92

Digel S., de Geus E., Thaddeus P., 1996, ApJ 422, 92

Dobashi K., Bernard J.-P., Yonekura Y., Fukui Y., 1994, ApJS 95,419

Dobashi K., Bernard J.-P., Fukui Y., 1996, ApJ 466, 282

Dorschner J., Gürtler J., 1964, Astron. Nachr. 297, 257

Han S.-T., Lee C.-H., Kim H.-R., Park D.-C., 1995, Int. J. IR \& MM Waves 17(1), 105

Heyer M.H., Vrba F.J., Snell R.L., et al., 1987, ApJ 321, 855

Heyer M.H., Carpenter J.M., Ladd E.F., 1996, ApJ 463, 630

Heyer M.H., Brunt C., Snell R.L., et al., 1998, ApJS 115, 241 
Huang Y.-L., 1986, Ph.D. Dissertation, Columbia University, NY, U.S.A.

Kawamura A., Onishi T., Yonekura Y., et al., 1998, ApJS 117, 387

Kutner M.L., Ulich B.L., 1981, ApJ 250, 341

Langer W., Wilson R.W., Goldsmith P.F., 1989, ApJ 337, 355

Laureijs R.J., Fukui Y., Helou G., et al., 1995, ApJS 101, 87

Lee Y., Snell R.L., Dickman R.L., 1990, ApJ 355, 536

Lee Y., 1994, JKAS 27(2), 147

Lee Y., Chung J.-H., Kim H.G., 1997, PKAS 12(1), 185

Lequeux J., Allen R.J., Guilloteau S., 1993, A\&A 280, L23

May J., Bronfman L., Alvarez H., Murphy D.C., Thaddeus P., 1993, A\&AS 99, 105

May J., Alvarez H., Bronfman L., 1997, A\&A 327, 325

Mead K.N., Kutner M.L., 1988, ApJ 330, 399

Moffat A.F.J., FitzGerald M.P., Jackson P.D., 1979, A\&AS 38, 197

Mooney T., 1992, Ph.D. Dissertation, University of New York. Stony Brook, U.S.A.
Racine R., 1968, AJ 73(4), 233

Sanders D.B., Clemens D.P., Scoville N.Z., Solomon P.M., 1986, ApJS 60, 1

Scoville N.Z., Yun M.S., Clemens D.P., Sanders D.B., Waller W.H., 1987, ApJS 63, 821

Sharpless S., 1959, ApJS 4, 257

Snell R.L., Heyer M.H., Schloerb F.P., 1989, ApJ 337, 739

Solomon P.M., Rivolo A.R., Barret J., Yahil A., 1987, ApJ 319, 730

van den Bergh S., 1966, AJ 71(10), 990

Usuda K.S., Hasegawa T., Handa T., et al., 1998, in The Physics and Chemistry of the Interstellar Medium, Abstract Book of the 3rd Cologne-Zermatt Symposium, Zermatt, September 22-25, 1998, V. Ossenkopf (ed.). Shaker-Verlag Aachen

Weaver H.F., Williams D.R.W., 1973, A\&AS 8, 1

Yonekura Y., Dobashi K., Mizuno A., Ogawa H., Fukui Y., 1997, ApJS 110, 21 